\title{
Décadrages Décadrages
}

cınéma, a travers champs Cinéma, à travers champs

1-2| 2003

Le hors-champ

\section{Et pour quelques notes de plus...}

\section{Louis Seguin}

\section{OpenEdition}

Journals

Édition électronique

URL : http://journals.openedition.org/decadrages/592

DOI : $10.4000 /$ decadrages.592

ISSN : 2297-5977

\section{Éditeur}

Association Décadrages

\section{Édition imprimée}

Date de publication : 1 octobre 2003

Pagination : 121-125

ISSN : 2235-7823

\section{Référence électronique}

Louis Seguin, «Et pour quelques notes de plus... », Décadrages [En ligne], 1-2 | 2003, mis en ligne le 26 avril 2013, consulté le 30 avril 2019. URL : http://journals.openedition.org/decadrages/592 ; DOI :

$10.4000 /$ decadrages.592 
Donc - c'est une conclusion mais provisoire, toujours à réviser, jamais forclose, rejetée, toujours béante, car tout laisse à prévoir qu'on n'en aura jamais fini de repasser par l'ouverture, de répéter le scandale de l'ingérence - il faut, puisque Leo Ramseyer y invite, revenir "encore» sur le "hors-champ", quitte à changer de ton. "Réfléchir les miroirs", comme y invitait (non, ce n'est pas Jean Cocteau) Jacques Rigaut. Car le chemin de la pensée ne passe pas par l'accumulation du «davantage» mais par le retournement, la révolution. Ce «désir» de révolution qui est si insupportable aux contempteurs essoufflés de la "pensée 68 ", les Luc Ferry et autres Jean Clair.

Donc, le cinéma invente son propre espace, sans avoir à puiser dans l'univers bien organisé que nous aurait fourni, clé en main, la Création. C'est, plus précisément, un espace qui lui est propre, non pas parce qu'il l'aurait acquis, qu'il aurait sur lui un droit de propriété, mais parce qu'il l'appréhende. L'invention doit être prise au pied de sa lettre. Elle est le «invenire» du latin, elle y vient, elle s'en mêle. Il y a, dans l'histoire de la philosophie, deux espaces parallèles et dont le parallélisme pose d'autres questions. L'un qui est placé du côté de la science appliquée et de la technique, de la géographie, de l'arpentage d'une terre que l'on peut mesurer et dont on peut faire commerce, qui se calcule et qui s'échange, où l'on peut se déplacer et que l'on peut connaître, qui a ses lois et sa triangulation et puis l'autre, qui est du côté de l'origine. Edmund Husserl, dans L'Origine de la géométrie: "Nous comprenons [...] son mode d'être persistant: il ne s'agit pas seulement d'un mouvement procédant d'acquis en acquis, mais d'une synthèse continuelle en laquelle tous les acquis persistent dans leur valeur, forment une totalité, de telle sorte qu'en chaque présent l'acquis total est, pourrait-on dire, prémisse totale pour les acquis de l'étape suivante. "1 Ou bien encore, un degré plus avant, c'est-à-dire plus loin, avec Martin Heidegger: "C'est seulement parce que l'être est dévoilé qu'il devient possible à l'existant de se manifester. »2 La philosophie pose et repose sans cesse la question et le Dieu créateur a été inventé pour ne plus avoir à la poser, pour l'effacer
1E. Husserl, L'Origine de la géométrie, Presses universitaires de France (Epiméthée), Paris, 1999, p. 177 (introduction de Jacques Derrida).

2 Martin Heidegger, "De l'essence du fondement", Questions I, trad. Henri Corbin, Gallimard (Classiques de la philosophie), Paris, 1987, p. 97. 
3 Friedrich Hölderlin, Douze poèmes, trad. et présentation de François Fédier, La Différence (Orphée), Paris, 1989, p. 55.

4 Hérodote, Histoires, II, 109, Les Belles lettres (Collection des universités de France), Paris, 1948, p. 137.

5 Martin Heidegger, L'art et l'espace, trad. Jean Beaufret et François Fédier, Erker-Verlag, St-Gall, 1983 (2 $2^{\text {ème }}$ éd.), p. 23. du tableau. Dieu console la pensée de sa paresse. Le hors-champ est une manière, au cinéma, de ne plus (se) poser la question dans le moment même où elle se pose, où André Bazin l'a posée.

L'espace du cinéma est une tentative pour la réitérer, pour maintenir la béance de la porte contre la pression du Créateur qui pousse pour la refermer. Il s'agit de savoir que cette question se pose derrière toute autre question, qu'il s'agisse du cadre, du champ et de la profondeur, de l'image-mouvement ou de l'image-temps. Et les grands cinéastes sont ceux-là qui (se) posent, dans chacun de leurs films, la question de l'apparition et de son émerveillement premier. Ou bien, si l'on préfère relire Tout comme au jour de fête... : "L'ivresse à nouveau se sent, / Elle, la Toute-Créatrice, encore une fois.»3 L'ivresse de Noé était un défi au "Père tout puissant, créateur du ciel et de la terre». Ou bien, si l'on préfère, la détresse et l'imploration vengeresse de l'orphelin.

L'Homme, en inventant Dieu, lui a offert la demeure d'un espace sans limites, la «robe sans couture», indéchirable, de l'infini et de l'éternité dont parle l'évangéliste Jean $(19,23)$. Et puis, de l'autre côté, dans la proximité d'un matérialisme païen, il y a les dieux trop humains et l'historien Hérodote qui explique dans ce qu'il appelle une "parenthèse" (une "parenthèkè", une digression si l'on veut) que ce sont les Egyptiens qui ont appris à mesurer la surface de leurs champs pour métrer ce qui éventuellement avait été emporté par les crues du Nil et pour ne pas avoir à payer les impôts fonciers afférents: "Il me semble que c'est de là que provient l'invention de la géométrie que les Grecs ont ensuite récupérée.»4 L'origine de la géométrie se confond-t-elle avec l'invention du cadastre? Le cinéma n'échappe pas à la contradiction; il est lui aussi coincé entre la fiscalité, l'arpentage, la machine de Brunelleschi, les traités de Girard Desargues sur la perspective et l'hypothèque divine du «hors-champ». Où en est, dans cette aporie de la pratique et de la théorie, le «réel» qui se filme? Du côté de la théologie? Du côté d'un matérialisme engelsien, d'une Dialectique de la nature? Ou si l'on préfère, comment la géométrie peut-elle être, comme dit Emmanuel Kant, "la science de toutes les espèces possibles d'espaces»?

Martin Heidegger, dans L'art et l'espace:

"L'art comme plastique: non une prise en main de l'espace. La sculpture ne serait pas un débat avec l'espace.

La sculpture serait alors une incorporation des lieux qui, ouvrant une contrée et la prenant en garde, tiennent rassemblés autour d'eux quelque chose de libre qui accorde à toute chose séjour et aux hommes habitation au milieu des choses» $\mathbf{5}$.

Pas de début, pas de création, pas d'intervention divine, pas de loi. Mais comment, au cinéma, le film s'incorpore-t-il dans l'ouverture de 
l'écran, dans cet espace dont il se saisit, qui ne peut appartenir de droit à personne, et surtout pas à un Créateur suprême, mais où il se donne quand il se projette. Comment s'installe, habite, une politique des auteurs, autrement dit, pour les cinéastes, une manière d'exiger un droit de cité, d'être les citoyens de cette polis, les sujets de cette administration et de sa profondeur6 ? Serge Daney et Jean-Claude Biette, dont l'absence se fait également sentir, s'obstinaient (c'est le rôle que cherche à tenir, difficilement, la critique) à découvrir dans les films, une expérience vécue, rêvée ou inventée du monde. L'«expérience», ici, n'a rien à voir avec la déduction et l'enseignement. Elle est cette aptitude singulière de l'entendre, d'arracher pour le replacer ce qui s'installe, comme lorsque Friedrich Nietzsche dit que «la vie pourrait être une expérience de celui qui cherche la connaissance». Car c'est bien là la question que pose, par défaut, dans sa marge, la "théorie du hors-champ", de cet espace qui n'existe pas davantage qu'il n'y avait de temps avant le big-bang, avant l'explosion et son vacarme.

À commencer, justement, par le son. Y a-t-il une localisation du son? Le son est-il assigné à résidence sur l'écran? Autrement dit, relève-t-il, comme le décor et les comédiens, d'une mise en scène? Le problème a été longuement discuté et ses conclusions définies, en particulier par Michel Chion7, mais il faut bien conclure que tous les effets d'«élargissement» du son rejoignent les effets d'«élargissement» de l'image. Si distendu soit-il, le champ sonore épouse le destin du champ visuel; il reste confiné dans ce qu'il élargit. Là encore, on peut relire Martin Heidegger.

Le son est la négation constante du hors-champ. Même si l'on ne voit pas la personne qui parle, ou qui chante, ou encore ce qui fait du bruit ou de la musique, le son ne peut passer que par l'écran. Quel que soit son appareillage (enceintes multiples ou écouteurs), il vient toujours de là. Il est toujours lié à la présence. Il est toujours monophonique, et c'est bien pourquoi Jean-Marie Straub et Danièle Huillet, même pour leur usage personnel, refusent la stéréo. Il est enfermé dans la solitude de la projection. À caméra unique, micro unique, quitte comme Louis Hochet à aller chercher dans les caves de la Radio de Francfort le micro indispensable que les progrès de la technique avaient rendu obsolète. C'était toutefois avec cet instrument d'un autre âge qu'il allait pouvoir enregistrer à la perfection, "épaisseur» incluse, la musique et les paroles de Du jour au lendemain d'Arnold Schönberg, y compris, ce qui n'est pas rien, lorsque les musiciens s'accordent avant le générique et qu'il y a encore du "désordre», puisque Michael Gielen 8 n'en a pas encore pris la «direction». C'est là, dans ce «là» que le cinéma se donne le «la»,
6 Hubert Damisch: "La perspective "centrale, comme déjà la géométrie grecque, aura été travaillée dès l'origine par la question de l'infini, que l'infini, dès l'origine, y aura fait irruption et cela au lieu, au point même du sujet." (H. Damisch, "La fissure", in Filippo Brunelleschi, 1377-1446. La Naissance de l'architecture moderne, Direction de l'architecture (L'Equerre), Paris, 1980, p. 35).

70n lira avec profit ses livres: La voix au cinéma, Editions de l'Etoile (Essais), Paris, 1982; La toile trouée ou la parole au cinéma, Cahiers du cinéma (Essais), Paris, 1988; L'audio-vision, Nathan (Cinéma-image), Paris, 1990; Le Son, Nathan (Cinéma-image), Paris, 1998.

8 II faudra que Gielen fasse un second enregistrement plus conforme aux normes du commerce lorsqu'il s'agira d'éditer un disque. 
9 Jean-Pierre Coursodon et Bertrand Tavernier, 50 ans de cinéma américain, Nathan, Paris, 1991, p. 445.

10 Georges Duby, Saint Bernard, l'art cistercien, Flammarion (Champs, $\mathrm{n}^{\circ} 77$ ), Paris, 1988 , p. 103. qu'il apprend que le son, et à plus forte raison la musique, n'ont pas de hors-champ. Le bruit, la voix ou la mélodie ont des sources et ils y sont liés. Ils passent par les corps, les objets ou les instruments. On ne s'y baigne pas, on n'y plane pas comme voudrait le faire croire l'imbécillité contemporaine, on les écoute. Le reste, c'est du commerce et la voix du Père éternel qui résonne sur l'univers. Dieu est un représentant en sound system.

Il faudrait voir et revoir un film de John Farrow (c'était, selon Jean-Pierre Coursodon et Bertrand Tavernier, son préféré) qui vient de repasser à la télévision, sur "Ciné-Classic», Alias Nick Beal, de 1949, dont le titre a été traduit par Un pacte avec le diable, très maladroitement parce que le nom du Malin n'est jamais prononcé et qu'il y a juste une allusion à son pseudonyme Old Nick. Jean-Pierre Coursodon et Bertrand Tavernier écrivent: "La mise en scène de Farrow [...] utilise une multitude de variations sur les entrées et les sorties (toujours insolites mais jamais délibérément «surnaturelles») de Ray Milland. "9 Le tentateur, en fait, surgit de nulle part; c'est un être infernal, il sort directement de l'enfer sans s'être faufilé dans un repli de la Tunique. Il n'entre même pas dans le champ; c'est le champ qui vient à lui et qui achoppe sur sa présence, il n'est pas là, dans les limbes, à attendre qu'on l'appelle. L'Ile des âmes perdues, où il entraîne ses victimes, ne figure sur aucune carte. Elle est là ; quelque part, dans la profondeur de l'écran ou dans l'abîme de son pourtour, au-delà du brouillard qui a envahi la toile. Parabole de la situation des comédiens: ils sont là, debout, les damnés de la terre, et ils payent cher ce droit d'être là. Ils n'ont pas d'espace légitime, ils ne sont pas des héritiers, et se pose alors, contre les hypothèses apaisantes du hors-champ où ils vivraient des jours tranquilles en attendant qu'on mette fin à leur intermittence, la question de leur droit au logement.

Dans le même esprit, on peut donc parler de la «fenêtre chez Jean Renoir» chère à Jean Douchet ou des portes chez Ernst Lubitsch. Elles s'ouvrent dans et sur la demeure de l'écran dont elles aménagent et redoublent l'accès, mais elles ne peuvent rien contre la menace qui pèse sur le plan, cerné par la forêt épaisse où il s'est ouvert une clairière. Il y a dans le cinéma une trace de l'épopée cistercienne: "Puisque...", écrit Georges Duby, "la morale de Saint Bernard s'enracine dans une méditation sur l'incarnation, de même le bâtiment cistercien commence à l'écran de sauvagerie que le monastère autour de lui protège. Il a pris corps au sein de cette enveloppe broussailleuse $\mathbf{1 0}^{\mathbf{1 0}}$. Le corps du Christ contre la Tunique? "Ecce homo"? Le mythe du Fils humilié et torturé contre le mythe du Père tout-puissant et triomphant? Et qui donnerait 
une autre image, "humaine» celle-là, écartelée, rapiécée, couverte de blessures, de cicatrices, de "sutures» qui seraient le prix de l'appropriation, la monnaie dont se paye, par le Rachat, le lieu que l'homme doit habiter de plein droit, poétiquement? Cet habitat, cette colonisation si l'on veut, ouvre d'autres questions, multiples. Jean-Pierre Oudart en a déjà posées $\mathbf{1 1}$ certaines. Mais rien n’interdit d'y revenir.

11 Jean-Pierre Oudart, "La suture", in Cahiers du cinéma, $\mathrm{n}^{\circ} 211$ et 212, 1969.

Vendredi $1^{\text {er }}$ août 2003 